\title{
Exploring microwave absorption by non-periodic metasurfaces
}

\author{
J. K. Hamilton ${ }^{1}{ }^{2}$, I. R. Hooper ${ }^{1}$, and C. R. Lawrence ${ }^{2}$ \\ ${ }_{1}^{1}$ Department of Physics and Astronomy, University of Exeter, Exeter, Devon, EX4 4QL, UK \\ ${ }^{2}$ QinetiQ Ltd, Cody Technology Park, Farnborough, GU14 OLX, UK \\ Corresponding author: J. K. Hamilton (e-mail: jkhamilton@qinetiq.com)
}

\begin{abstract}
In recent years there has been a large body of work investigating periodic metasurface microwave absorbers. However, surprisingly few investigations have focused on the absorption performance of similar non-periodic designs. In this work, the electromagnetic response of a large area $(310 \mathrm{~mm} \times$ $310 \mathrm{~mm}$ ) microwave absorber that lacks a global periodicity is experimentally studied. The top metallic layer of the ultra-thin $(0.3 \mathrm{~mm})$ absorber is structured with rectangular patches given by a procedurally generated non-periodic pattern, known as the toothpick sequence. The specular reflectivity of both $p$ polarised and s-polarised incident radiation shows coupling to an additional low frequency mode when compared to a standard square patch periodic absorber. To further explore the coupling efficiency of such non-periodic absorbers, finite element models were used to investigate the influence of increasing sample size.
\end{abstract}

INDEX TERMS Non-periodic, microwave absorber, metasurface.

\section{INTRODUCTION}

I $\mathrm{N}$ recent years there has been a large amount of interest in RF absorbers based on metamaterials. [1], [2] The majority of these structures rely on periodically arranged unit cells with predesigned dimensions. These periodic metamaterials have a wide range of potential applications including radar cross-section (RCS) reduction, [3] sensors, [1], [4] and solar cells. [1], [5] Within this field, research has largely focused on periodic metallic structures on metal-backed dielectrics, due to their simplicity, ease of fabrication, and performance.

One of the simplest examples of such an absorber is the work of Hibbins et al. (2004). [6] In their investigation, a structure composed of thin (18 micron) metallic strips separated from a ground plane by a thin dielectric was shown to be an incredibly effective narrow-band absorber. The resonance frequencies of the structure were simply determined by the width of the metallic strips, and the permittivity and thickness of the dielectric core. One limitation of this early design was that the structure could only selectively absorb one polarisation.

In subsequent years, these simple absorbers have been adapted to use a range of different periodic patterns, [7][11] whilst various efforts have been made to add additional resonances or to broaden the bandwidth, for example by using multi-layer structures [12] or multi-resonant unit cells. [13]-[15]

Multi-layer structures are an extremely effective method for broadening the absorption bandwidth. However, the broadening of the modes comes at the expense of increasing the total thickness of the absorber, which may be unde- sirable for certain applications. One example of a multilayer system involves a unit cell made up of a stack of 20 metallic patches, reducing in size towards the top of the stack, separated by dielectric layers. [12] It was shown that by using such a stacked structure, it was possible have an absorption level around $90 \%$ for a frequency range of 7.6 $\mathrm{GHz}$ to $17.4 \mathrm{GHz}$.

Another interesting method for broadening the absorption band is to create a unit cell with multiple resonant structures. [14] It has been shown that a unit cell made up of four metallic strips with increasing width is capable of increasing the bandwidth by three times that of the single strip resonator.

More recently, absorbers using fractal geometries have been proposed as a route to multiple frequency responses. A fractal is a never-ending pattern; they are created by repeating a simple process over an ongoing feedback loop. Fractals are commonly used in antenna design to maximise the effective length of the materials used. [16] In Venneri et al. (2019), [17] the unit cell consisted of four fractal cells to create a dual-frequency response. The advantage of such a structure is the addition of multiple resonances without changing the overall size of the fractal cells. Whilst other research has been conducted on fractal absorbers, [18]-[21] the majority still rely on periodically repeating a fractal unit cell.

Interestingly, despite the focus on periodic arrays of fractal patterns, little research has been conducted on structures that lacks any global periodicity. Such structures are also commonly known as non-periodic structures. In Yang et 
al. (2017) [22] absorbers using a unit cell (2.5 times the design wavelength) arranged in Fibonacci and Thue-Morse lattices were investigated, with the non-periodic lattices having a reduced total number of unit cells compared to the periodic case. It was shown that the absorption behaviours of these non-periodic structures were very similar to those of their periodic counterparts. In addition to these investigations, there has been interest in creating programmable metasurfaces to tune the scattering properties of disordered structures. [23]

Here, we experimentally study the electromagnetic response for both $p$-(TM) and $s$-(TE) polarised radiation of a large-area, ultra-thin absorber based on non-periodic 2dimensional mathematical sequences. We consider the previously well-studied square patch array case [8] and compare the response with two samples based on the mathematical sequence known as the toothpick sequence. [24] Finite element modelling (using COMSOL Multiphysics) is also used to help understand the behaviour of these non-periodic structures.

\section{METHOD}

The toothpick sequence is a complex 2-dimensional mathematical sequence [24]. This pattern is formed by repeatedly adding line segments - known as toothpicks - to the free ends of the previous pattern in the sequence. For example, the initial pattern in the sequence $(n=1)$ would be a single toothpick. The next pattern $(n=2)$ would add two toothpicks perpendicular and centred on each end of the previous. This sequence is of interest due to it being mathematically generated [24] and that the pattern naturally produces square and rectangular patches of varying size. Fig. 1 shows an example of this growth pattern up to $n=7$, as well as an example of $n=64$. The red toothpicks are those that were added in the stated stage of the sequence.

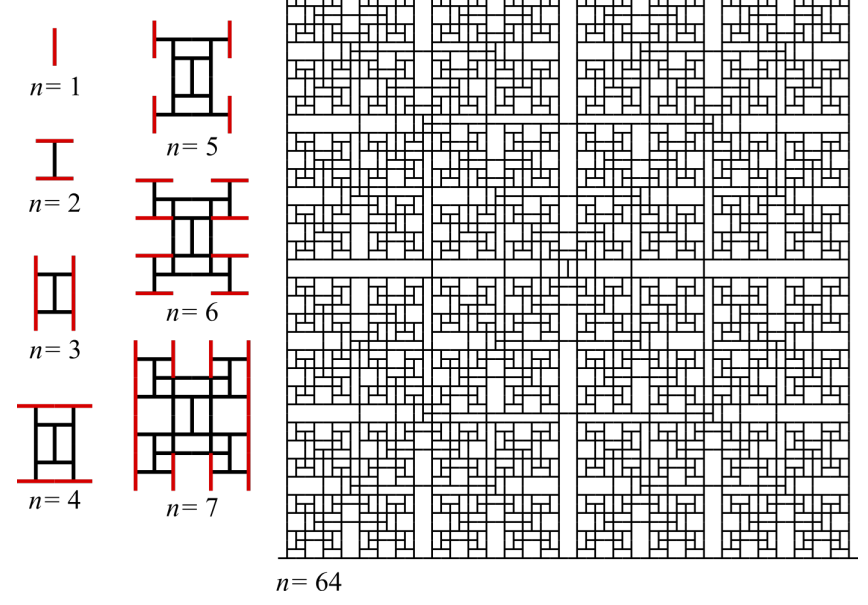

FIGURE 1. An example of the toothpick sequence growth up to $n=7$ (the unit cell used for TPn7). The red toothpicks show the added segments for the current stage of the sequence. In addition $n=64$ is also shown.

Here, we experimentally investigate the electromagnetic response of three ultra-thin (relative to the wavelength) absorbers consisting of a perforated and solid metallic layer $\left(t_{\mathrm{Cu}}=35 \mu \mathrm{m}\right.$ copper) separated from a ground plane $\left(t_{\mathrm{Cu}}=35 \mu \mathrm{m}\right.$ copper) by a dielectric layer (glass-reinforced epoxy laminate material - known as FR4) $\left(t_{\mathrm{d}}=0.3 \mathrm{~mm}\right)$. The first design consists of a periodic square patch array, based on the work of Brown et al. (2008). [8] This has a unit cell size of $G=10 \mathrm{~mm}$ and slit widths of $w_{\mathrm{s}}=0.3 \mathrm{~mm}$, giving a resonance frequency of $\sim 7 \mathrm{GHz}$ for its fundamental mode (see the schematic of the cross section in Fig. 2(a)). This periodic structure is well-studied within the literature and is used here for comparison purposes. The two other samples were variants based on the toothpick sequence. The first consists of a periodic array of a unit cell of $n=7$. The dimensions of the unit cell were $30 \mathrm{~mm} \times 40 \mathrm{~mm}$. The unit cell contained eight $G / 2$ square patches, four $G$ square patches, and twelve $G / 2$ by $G$ rectangular patches. The final sample was a fully non-periodic structure, resulting from the toothpick sequence of $n=64$. This sample was designed to have no global periodicity. For both toothpick samples the toothpick length was $G=10 \mathrm{~mm}$ and width 0.3 $\mathrm{mm}$, resulting in the smallest metallic patch size being $G / 2$. Photographs of central regions of each sample are shown in Fig. 2(b-d). Each sample had overall dimensions of $310 \mathrm{~mm}$ $\times 310 \mathrm{~mm}$, and we refer to each of the toothpick samples as: toothpick $n=7$ (TPn7), and toothpick $n=64$ (TPn64).
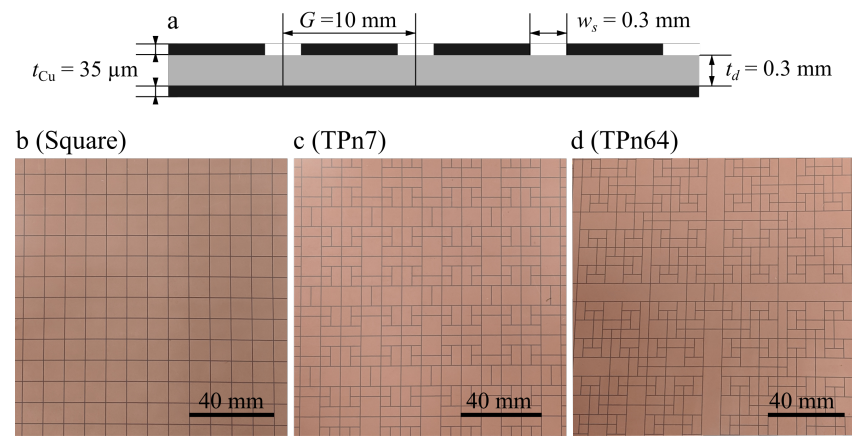

FIGURE 2. Examples of the fabricated samples. (a) A schematic of the cross-section through the periodic square patch array. Photographs showing (b) square, (c) toothpick $n=7$ (TPn7), and (d) toothpick $n=64$ (TPn64).

The specular reflectivity was measured as a function of elevation $(\theta)$ and azimuth $(\phi)$ angles for both $p$-(TM) and $s$-(TE) polarised radiation. To conduct the measurements a Naval Research Laboratory (NRL) arch was used. A NRL arch consists of two microwave horn antennas both connected to a vector network analyser (VNA) within an anechoic chamber. The reflection response was measured for all samples, as well as their associated phases. Fig. 3 shows a schematic of the NRL arch setup. The elevation angle of incidence measurements were swept between $10^{\circ}$ and $60^{\circ}$ from normal (in $5^{\circ}$ steps), with a fixed azimuth angle of $0^{\circ}$. The azimuth angle measurements were swept between $0^{\circ}$ and $90^{\circ}$ (in $10^{\circ}$ steps), with a fixed elevation angle of $60^{\circ}$. 


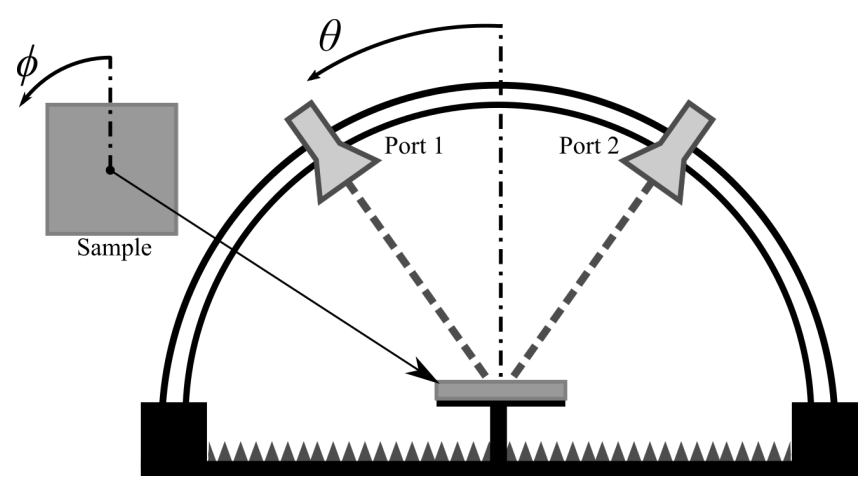

FIGURE 3. Schematic of the experimental setup. The horn antennas labelled Port 1 and Port 2 - were connected to a vector network analyser. The angles of $\theta$ and $\phi$ are also shown on the diagram.

\section{RESULTS}

\section{A. SQUARE PATCHES}

Fig. 4(a) shows the reflectivity as a function of elevation at a fixed azimuth angle of $\phi=0^{\circ}$ for $p$-polarised radiation. The dark bands indicate strong absorption, with the fundamental mode of the structure at $6.9 \mathrm{GHz}$ and the second order mode at $13.7 \mathrm{GHz}$.
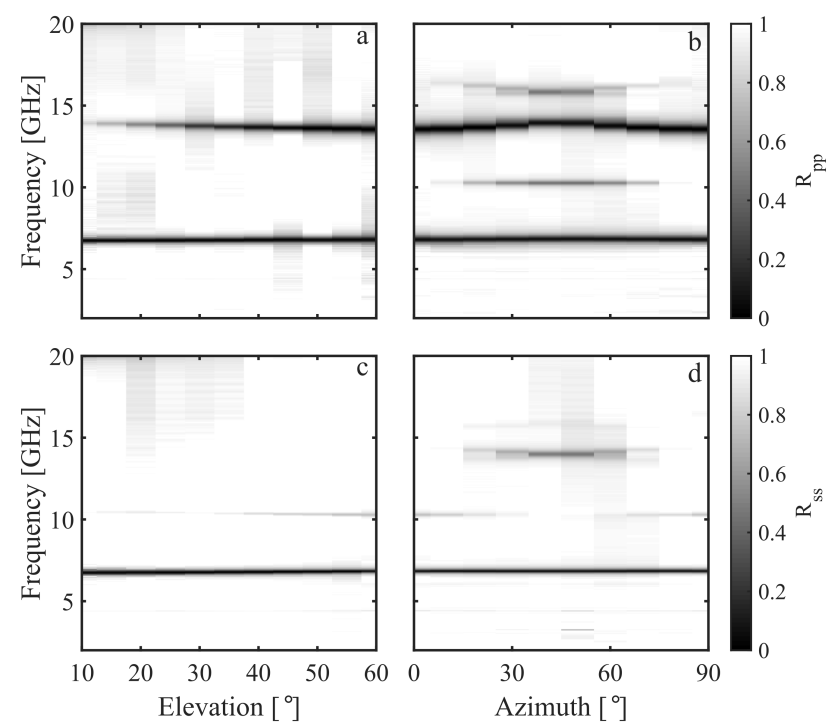

FIGURE 4. Experimental $R_{\mathrm{pp}}$ and $R_{\mathrm{ss}}$ data for the square patch sample. (a) $R_{\mathrm{pp}}$ as a function of elevation angle of incidence, at $\phi=0^{\circ}$. (b) $R_{\mathrm{pp}}$ as a function of azimuth angle, at $\theta=60^{\circ}$. (c) $R_{\mathrm{SS}}$ as a function of elevation angle of incidence, at $\phi=0^{\circ}$. (d) $R_{\mathrm{SS}}$ as a function of azimuth angle, at $\theta=60^{\circ}$.

The fundamental resonance corresponds to a halfwavelength fitting in the dielectric beneath each patch, with field maxima at either end of the patch and a null in the centre. Due to the thinness of the dielectric this field is highly localised with very little coupling between patches. As such the mode is remarkably non-dispersive even up to high elevation angles. The second order mode corresponds to a whole wavelength fitting beneath each patch, but due to symmetry this cannot be excited by a normally incident $\left(\theta=0^{\circ}\right)$ plane wave. As the elevation angle of incidence is increased a phase shift along the surface is created, enhancing the coupling to the even-numbered harmonics. As a result, the second mode is strongest at large angles of elevation and the intensity reduces as the incident wave becomes closer to normal incidence $\left(\theta=0^{\circ}\right)$.

Fig. 4(b) shows the specular reflection as a function of azimuth angle while keeping the elevation angle at $\theta=60^{\circ}$ (chosen so as to ensure strong coupling to both the fundamental and second order modes). The frequency of the fundamental mode remains constant at $6.9 \mathrm{GHz}$ regardless of the azimuth angle, whilst the second order mode shows some degree of curvature. The additional modes shown at 10.3 $\mathrm{GHz}$ and between $15.0 \mathrm{GHz}$ and $16.5 \mathrm{GHz}$ are due to the phase reversal across the patch driving the charges of like-sign to diagonally opposing corners, permitting coupling. The curvature of the mode between $15 \mathrm{GHz}$ and 16.5 $\mathrm{GHz}$ is due to the change in momentum available as the sample is rotated. [8]

Fig. 4(c) shows the reflectivity for $s$-polarised radiation. Similar to $R_{\mathrm{pp}}$, coupling to the fundamental mode at $6.9 \mathrm{GHz}$ is allowed. However, the second harmonic coupling is not possible for $\phi=0^{\circ}$. The $\pi$-phase shift within the structure results in opposite phases at adjacent slits, meaning that coupling to the mode at $13.7 \mathrm{GHz}$ is not possible.

Fig. 4(d) shows that the $13.7 \mathrm{GHz}$ mode has an angle dependent coupling strength for $s$-polarised radiation. This angle dependence results in a loss of coupling between azimuthal angles of $0^{\circ}$ and $20^{\circ}$ as well as between $70^{\circ}$ and $90^{\circ}$ - due to symmetry. It is also evident that for this polarisation, there is weak coupling to the $10.3 \mathrm{GHz}$ and $16.4 \mathrm{GHz}$ modes.

\section{B. TOOTHPICK SEQUENCE}

Fig. 5(a-b) shows $R_{\mathrm{pp}}$ as a function of elevation and azimuth angle for the TPn7 sample. As previously discussed, this sample uses a periodic arrangement of the $n=7$ toothpick sequence. Fig. 5(a) shows that the fundamental 6.9 $\mathrm{GHz}$ mode is present for all angles, similar to the square patch design. However, the second harmonic (13.7 $\mathrm{GHz}$ mode) shows an angle-independent response, as well as a broadening of the mode. By using the full width at half maximum (FWHM), the bandwidth - averaged over the measured range - is $1.2 \mathrm{GHz}$ with a standard deviation of $0.2 \mathrm{GHz}$, compared to the maximum bandwidth of $0.6 \mathrm{GHz}$ (at $\theta=60^{\circ}$ ) for the periodic square patch sample.

Fig. 5(b) shows coupling to the four modes as previously seen with the square patch sample. However, once again we observe a broadening of the second harmonic mode, which also reduces the relative effect of the mode curvature. There is an additional feature in the spectra between 14 and 15 $\mathrm{GHz}$ for angles of $\phi=0^{\circ}$ to $\phi=40^{\circ}$. This feature is due to the onset of a diffracted order - see Fig. 7(b) for all diffraction edges.

Fig. 5(c) shows $R_{\mathrm{pp}}$ for the TPn64 sample. The response is very similar to the TPn7 sample, with the fundamental mode at $6.9 \mathrm{GHz}$ and the broadened second harmonic. 

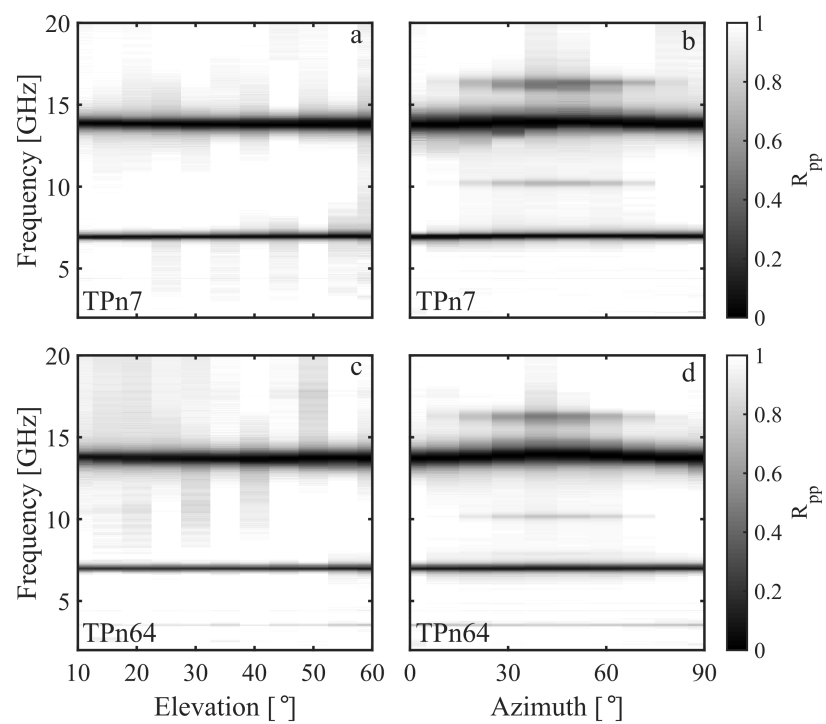

FIGURE 5. Experimental $R_{\mathrm{pp}}$ data as a function of elevation and azimuth angles of incidence. (a) TPn7 sample, elevation angle of incidence sweep with $\phi=0^{\circ}$. (b) TPn7 sample, azimuthal angle sweep with $\theta=60^{\circ}$. (c) TPn64 sample, elevation angle of incidence sweep with $\phi=0^{\circ}$. (d) TPn64 sample, azimuthal angle sweep with $\theta=60^{\circ}$.

For comparison, the bandwidth of the second harmonic is $1.0 \mathrm{GHz}$ with a standard deviation of $0.1 \mathrm{GHz}$. Fig. 5(d) again shows little difference between the TPn7 and TPn64 samples, apart from an additional weak mode, which can be seen at $3.5 \mathrm{GHz}$. Additional modes are to be expected due to there being a larger number of patch sizes available for excitation on the TPn64 sample. For example, the weak mode at $3.5 \mathrm{GHz}$ is due to coupling to the fundamental mode of patches with a side length of twice a standard square patch $(2 G)$.

It is also worth noting that the diffraction edge present in Fig. 5(b) is not present in the TPn64 sample. This is due to the structure lacking a global periodicity. As a result, the TPn64 sample has broadband non-dispersive modes that are unaffected by the onset of any coherent scattering.

Equivalent reflectivity data were also obtained for $s$ polarised radiation, the results of which are shown in Fig. 6. The main point of interest in Fig. 6(a) is the addition of the second harmonic $(13.7 \mathrm{GHz})$ mode previously seen in the $R_{\mathrm{pp}}$ data. This additional mode is also angle-independent across the full range of elevation angles. As previously discussed, the periodic square patch surface cannot support this mode when excited by $s$-polarised radiation due to the $\pi$-phase shift within the structure.

Fig. 6(b) shows the specular reflectivity as a function of azimuth angle while keeping the angle of incidence at a value of $\theta=60^{\circ}$. The TPn 7 sample not only allows coupling to the second harmonic over the full angle range, the mode is also broadened - similar to that seen for $R_{\mathrm{pp}}$.

The $R_{\mathrm{ss}}$ response for the TPn64 sample is shown in Fig. 6(c-d). The coupling to the second harmonic is present, and the response is similar to the TPn7 sample.
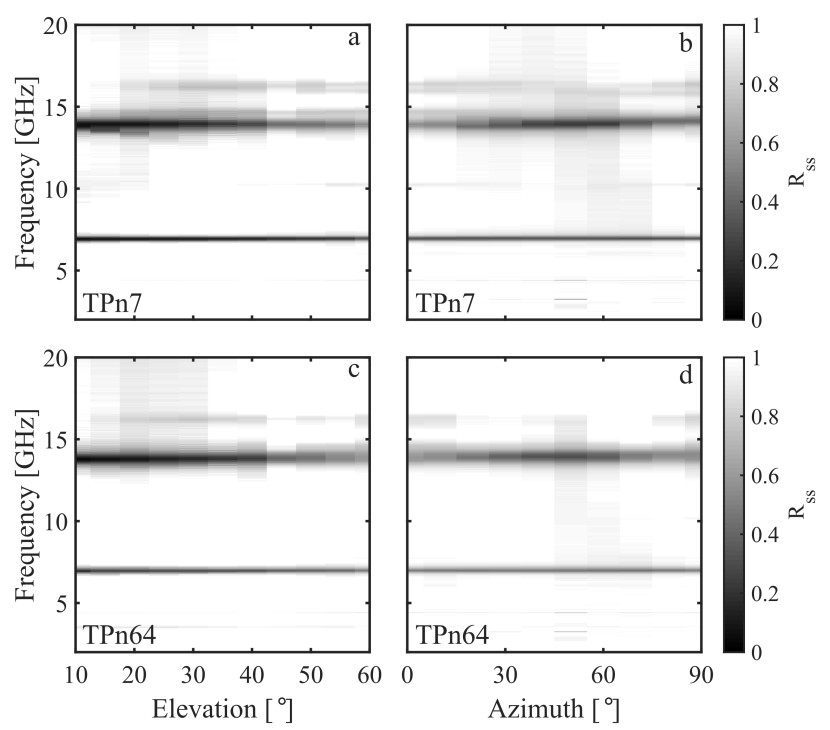

FIGURE 6. Experimental $R_{\mathrm{SS}}$ data as a function of elevation and azimuth angles of incidence. (a) TPn7 sample, elevation angle of incidence sweep with $\phi=0^{\circ}$. (b) TPn7 sample, azimuth angle sweep with $\theta=60^{\circ}$. (c) TPn64 sample, elevation angle of incidence sweep with $\phi=0^{\circ}$. (d) TPn64 sample, azimuth angle sweep with $\theta=60^{\circ}$.

\section{FINITE ELEMENT MODELLING}

From the above experimental results, it appears that the toothpick designs may give some advantages over the standard square patch absorbers; namely multi-banded absorption, and broader non-dispersive and polarisation insensitive absorption bands. However, from these experiments it cannot be determined whether there has been an increase in the absorption, or whether power might instead have been scattered into other radiative loss channels. To test this we turn to finite element modelling using COMSOL Multiphysics with the additional RF module.

In order to validate our models we initially focused on recreating our experimental results from the TPn7 sample. Due to this structure being periodic, one can model the geometry using a unit cell and periodic boundary conditions. The permittivity of the dielectric core was $\varepsilon=4.17-0.07 i$ (mimicking FR4), and for ease of modelling the two copper layers were modelled as infinitely thin perfect conductors.

The modelled response for $R_{\mathrm{pp}}$ as a function of azimuth angle, at $\theta=60^{\circ}$ is shown in Fig. 7(a), and compares well to the experimental data (Fig. 5(b)). In addition to obtaining the polarisation-conserved specular reflection, we can also calculate the power "lost" into all possible other radiative channels, whether that be into polarisation-converted radiation or into diffracted orders. The diffracted orders can be predicted by using the following equations

$$
k_{0}^{2}=k_{x}^{2}+k_{y}^{2}
$$

where

$$
k_{x}=k_{0} \sin \theta \cos \phi \pm M k_{g x},
$$


and

$$
k_{y}=k_{0} \sin \theta \sin \phi \pm N k_{g y},
$$

where $M$ and $N$ are the mode orders, $k_{g x}=\frac{2 \pi}{G_{x}}$, and $k_{g y}=$ $\frac{2 \pi}{G_{y}} . G_{x}$ and $G_{y}$ are the length of the unit cell in the xdirection and y-direction, respectively.

The sum of power lost into all possible other radiative channels is shown in Fig. 7(b), along with all of the diffraction edges for the various diffracted orders (since the period of the TPn7 design is much longer than the wavelength there are a large number of diffracted orders into which power could be lost). It is clear that, on-resonance, a large proportion of the power does go into these other radiation channels, especially for the mode at around 14 GHz.
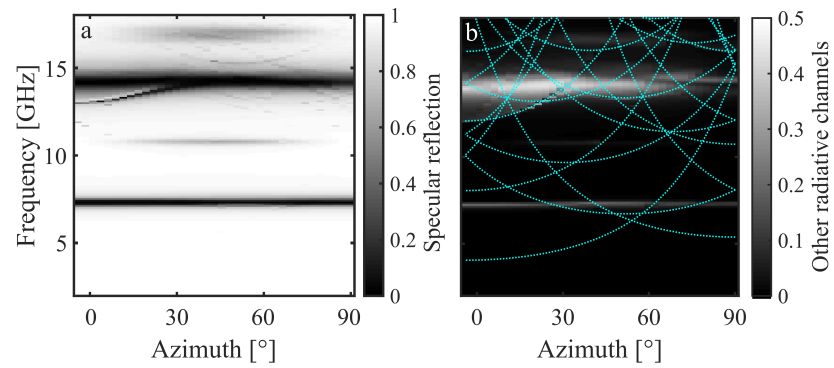

FIGURE 7. Modelled response of the TPn7 sample. (a) $R_{\mathrm{pp}}$ as a function of azimuth angle, at $\theta=60^{\circ}$. (b) Total power lost into all other radiative channels scaled between 0 and 0.5 . The blue dotted lines show the diffraction edges.

Due to the large dimensions of higher-order toothpick designs it is not feasible to model periodic arrays of such in the same way as above (similarly, it is not possible to model higher order finite-sized non-periodic systems such as our TPn64 design). However, in an attempt to shed further light on the behaviour of these structures, we can model the normal incidence response by using a quarter of a unit cell with appropriate boundary conditions. This then allows us, within our computational constraints, to model toothpick designs up to $n=15$. We can then investigate the proportion of the power going into all diffracted orders for increasing $n$, and also the amount of power being absorbed. This is shown in Fig. 8.
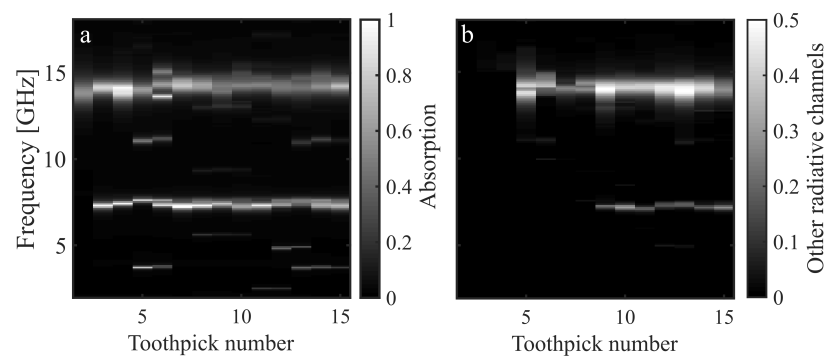

FIGURE 8. Normal incidence response as a function of frequency and increasing toothpick number at $\phi=60^{\circ}$. (a) The absorption and (b) the total power contained in all diffracted orders.
Unfortunately, for all toothpick designs where $n$ is sufficiently large that diffraction occurs, it is clear that a rather large proportion of power is being lost into diffracted orders rather than being absorbed. It is interesting to note, however, that all toothpick designs show absorption at the second harmonic $(13.7 \mathrm{GHz})$, even at normal incidence. For square patches at normal incidence, this second harmonic is not present for either polarisation.

\section{CONCLUSION}

In this work, the specular reflectivity of three metasurface designs was measured as a function of elevation and azimuth angles for both $p$ - and $s$-polarised incident waves. The main sample of interest was a patch array based on the toothpick sequence with $n=64$. This large-area sample lacked a global periodicity. The two other samples were a periodic version of the toothpick sequence - using a lower order as a unit cell - and a standard square patch array for comparison.

The standard square patch absorber has a single absorption band $(\sim 7 \mathrm{GHz})$ that is independent of elevation, azimuth and polarisation, plus an intermittent, polarisationsensitive, second band at $\sim 14 \mathrm{GHz}$. It was shown that the toothpick samples broadened the reflection minima in the specular response of the higher order modes for a $p$ polarised incident wave. For the case of the $s$-polarised incident wave, additional modes not present in the standard square array were excited.

To further investigate the potential use of the designs as absorbers, finite element models were used to calculate the proportion of the power lost into any non-specular orders. Whilst no clear relationship between toothpick number and the amount of power lost into diffracted orders was present, it was clear that a large proportion of power was indeed lost to these orders rather than being absorbed. It is not possible to draw a conclusion as to whether higher-order, non-periodic, toothpick designs, such as the TPn64 sample investigated here, would also suffer from scattering into nonspecular radiation. It would require substantially more complex experiments, or significantly increased computational power, to determine this.

\section{ACKNOWLEDGMENT}

This work was also supported by the UK Engineering and Physical Sciences Research Council Prosperity Partnership, TEAM-A (EP/R004781/1).

\section{REFERENCES}

[1] C. M. Watts, X. Liu, and W. J. Padilla. Metamaterial electromagnetic wave absorbers. Advanced materials, 24(23):98-120, 2012.

[2] L. B. Kong, Z. W. Li, L. Liu, R. Huang, M. Abshinova, Z. H. Yang, C. B. Tang, P. K. Tan, C. R. Deng, and S. Matitsine. Recent progress in some composite materials and structures for specific electromagnetic applications. International Materials Reviews, 58(4):203-259, 2013.

[3] B. A. Munk. John Wiley \& Sons, Ltd, 2000.

[4] A. Tittl, P. Mai, R. Taubert, D. Dregely, N. Liu, and H. Giessen. Palladiumbased plasmonic perfect absorber in the visible wavelength range and its application to hydrogen sensing. Nano letters, 11(10):4366-4369, 2011. 
[5] C. Bauer and H. Giessen. Light harvesting enhancement in solar cells with quasicrystalline plasmonic structures. Optics express, 21(103):A363A 371, 2013.

[6] A. P. Hibbins, J. R. Sambles, C. R. Lawrence, and J. R. Brown. Squeezing millimeter waves into microns. Phys. Rev. Lett., 92:143904, 2004.

[7] N. I. Landy, S. Sajuyigbe, J. J. Mock, D. R. Smith, and W. J. Padilla. Perfect metamaterial absorber. Physical review letters, 100(20):207402, 2008.

[8] J. R. Brown, A. P. Hibbins, M. J. Lockyear, C. R. Lawrence, and J. R. Sambles. Angle-independent microwave absorption by ultrathin microcavity arrays. Journal of Applied Physics, 104(4):043105, 2008

[9] J. R. Brown. The microwave response of ultra thin microcavity arrays. $\mathrm{PhD}$ thesis, University of Exeter, 2010.

[10] J. R. Brown, A. P. Hibbins, C. R. Lawrence, M. J. Lockyear, and J. R. Sambles. Microwave resonances of ultrathin hexagonally symmetric microcavity arrays. Journal of Applied Physics, 112(1):014904, 2012.

[11] M. Camacho, A. P. Hibbins, and J. R. Sambles. Resonantly induced transparency for metals with low angular dependence. Applied Physics Letters, 109(24):241601, 2016.

[12] X. Lleshi, R. Grelot, T. Q. Van Hoang, B. Loiseaux, and D. Lippens. Wideband metal-dielectric multilayer microwave absorber based on a single step fdm process. 2019 49th European Microwave Conference (EuMC), pages 678-681, 2019.

[13] Z. Wei, H. Li, C. Wu, Y. Cao, J. Ren, Z. Hang, H. Chen, D. Zhang, and C. T. Chan. Anomalous reflection from hybrid metamaterial slab. Optics Express, 18(12):12119-12126, 2010.

[14] Y. Cui, J. Xu, K. Hung Fung, Y. Jin, A. Kumar, S. He, and N. X. Fang. A thin film broadband absorber based on multi-sized nanoantennas. Applied Physics Letters, 99(25):253101, 2011.

[15] J. Xu, R. Li, S. Wang, and T. Han. Ultra-broadband linear polarization converter based on anisotropic metasurface. Opt. Express, 26(20):26235$26241,2018$.

[16] D. H. Werner and S. Ganguly. An overview of fractal antenna engineering research. IEEE Antennas and propagation Magazine, 45(1):38-57, 2003

[17] F. Venneri, S. Costanzo, and A. Borgia. A dual-band compact metamaterial absorber with fractal geometry. Electronics, 8(8):879, 2019.

[18] S. Costanzo, F. Venneri, G. Di Massa, A. Borgia, A. Costanzo, and A. Raffo. Fractal reflectarray antennas: state of art and new opportunities. International Journal of Antennas and Propagation, 2016, 2016.

[19] W. Zuo, Y. Yang, X. He, D. Zhan, and Q. Zhang. A miniaturized metamaterial absorber for ultrahigh-frequency rfid system. IEEE Antennas and Wireless Propagation Letters, 16:329-332, 2016.

[20] F. Venneri, S. Costanzo, and G. Di Massa. Fractal-shaped metamaterial absorbers for multireflections mitigation in the uhf band. IEEE Antennas and Wireless Propagation Letters, 17(2):255-258, 2017.

[21] Mitchell Kenney, James Grant, Yash D Shah, Ivonne Escorcia-Carranza Mark Humphreys, and David RS Cumming. Octave-spanning broadband absorption of terahertz light using metasurface fractal-cross absorbers. Acs Photonics, 4(10):2604-2612, 2017.

[22] Q. Yang, X. Chen, Y. Li, X. Zhang, Y. Xu, Z. Tian, C. Ouyang, J. Gu, J. Han, and W. Zhang. Aperiodic-metamaterial-based absorber. APL Materials, 5(9):096107, 2017.

[23] Mohammadreza F. Imani, David R Smith, and Philipp del Hougne. Perfect absorption in a disordered medium with programmable meta-atom inclusions. Advanced Functional Materials, 30(52):2005310, 2020.

[24] D. Applegate, O. E. Pol, and N. J. A. Sloane. The toothpick sequence and other sequences from cellular automata. arXiv preprint arXiv:1004.3036, 2010. 